\title{
Stercoral Perforation of the Rectum: A Case Report and Review of Literature
}

\author{
Mohammad M. Abbas ${ }^{1}$, MD, Aisha M. Abdulrahman ${ }^{2}$, MD, \\ Nouf Y. Akeel ${ }^{1}$, MD, and Majid M. Manouri' ${ }^{1}$, MD \\ ${ }^{1}$ Department of Surgery, Faculty of Medicine, King Abdulaziz University \\ ${ }^{2}$ Department of Surgery, East Jeddah Hospital \\ Jeddah, Saudi Arabia
}

\section{Correspondence}

Dr. Mohammad Abbas

Department of Surgery, Faculty of Medicine

King Abdulaziz University

P.O. Box 80215, Jeddah, 21589

Saudi Arabia

e.M: m.abbas1992@gmail.com

Submission: $\quad 30 \mathrm{Jul} .2018$

Accepted: $\quad 11$ Oct. 2018

\section{Citation}

Abbas MM, Abdulrahman AM, Akeel NY, Manouri MM. Stercoral perforation of the rectum: A case report and review of literature. JKAU Med Sci 2018; 25 (2): 51-56. DOI: 10.4197/ Med. 25.2.6

Copyright: @The Author(s), YEAR. Publisher. The Journal of King Abdualziz University - Medical Sciences is an Official Publication of "King Abdulaziz University". It is an open-access article distributed under the terms of the Creative Commons Attribution Non-Commercial License, which permit unrestricted non-commercial use, distribution, and reproduction in any medium, provided the original work is properly cited.
renser

\begin{abstract}
Stercoral perforation of the rectum is a rare disease. It is a potentially fatal complication of untreated chronic constipation. Presenting symptoms and signs are variable according to the site of the perforation. A 60-year-old male patient presented to the emergency room with dizziness and decreased level of consciousness. He was admitted for management under care of medical team as a case of urosepsis. Two days after admission he developed picture of Fournier gangrene. The underlying cause was found to be rectal perforation. Stercoral perforation is an emergency that can be missed if not highly suspected.We present a case of stercoral rectal perforation with picture of urosepsis and Fournier gangrene as presenting symptoms. Stercoral perforation is a potentially fatal disease with unspecific presentations that need to be highly suspected in patients with chronic constipation and severe sepsis.
\end{abstract}

\section{Keywords}

Stercoral; Constipation; Perforation; Peritonitis; Rectum

\section{Introduction}

S tercoral perforation of the rectum is a rare disease. The exact incidence is unknown, but it is thought to be under estimated. In a retrospective study in University of Berne, Switzerland, the incidence of colonic stercoral perforation was $3.2 \%$ of all colonic perforations ${ }^{[1]}$.

It is a potentially fatal complication of untreated chronic constipation. The overall mortality has been reported to be up to $34 \%^{[2]}$. The clinical presentation is variable according to the site of perforation, which results in a delay in the diagnosis and management.

We present a case of a rectal stercoral perforation of the rectum with an unusual presentation that was initially misdiagnosed as urosepsis.

\section{Case Report}

A 60-year-old male presented to the emergency department with a one-day history of altered level of consciousness, dysuria and back pain. There was no abdominal pain, however, he had chronic constipation. No history of rectal bleeding or rectal trauma. No history of rectal foreign body insertion. Also, no previous attempt was made to manually evacuate the rectum. The past medical history includes diabetes for 15 years and a recent stroke with minimal residual weakness. He had no previous surgeries. On examination, he was drowsy, severely dehydrated. He had a temperature of $38.3^{\circ} \mathrm{C}$ and pulse rate of 127 beat/minute. Cardiac, respiratory, and abdominal examinations were unremarkable. Digital rectal examination was not 
performed at the time. Laboratory work up revealed leukocytosis of 14 , elevated serum creatinine, high urea and glucose level with hyponatremia. Urine dipstick was positive for both glucose and nitrate.

The patient was admitted under care of medical team as a case of urosepsis. Urine and blood cultures were obtained. Urine culture showed Acinetobacter baumannii and blood cultures showed fungemia. The patient was started on intravenous antibiotics and fluids for rehydration. Initially, he responded to the management in form of stabilized vital signs and improved level of consciousness. Two days later, the patient started to complain of lower abdominal pain and he developed rapidly progressing genital ulcer associated with hemodynamic instability. His leukocyte

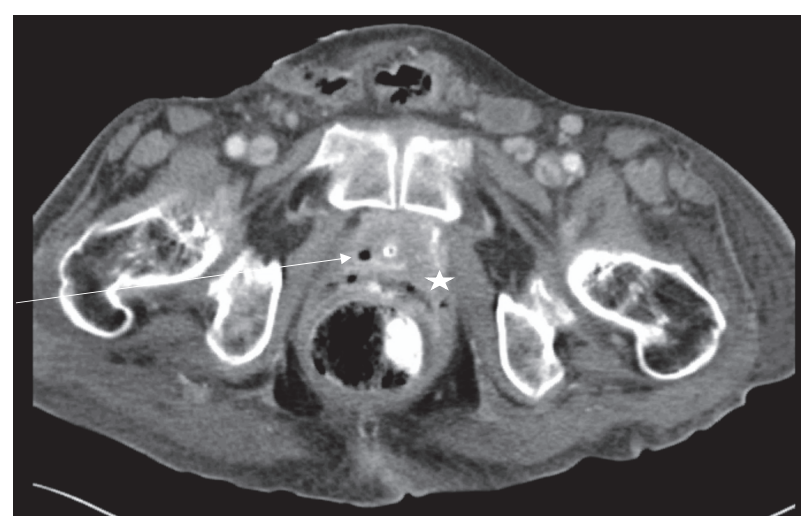

Figure 1. Computed tomography scan of the abdomen and pelvis with IV, oral and rectal contrast, axial section, showing contrast at the rectum and perineum, presence of air in the perineum, and abscess formation. Arrow showing air in the pelvis. Star showing contrast leak.

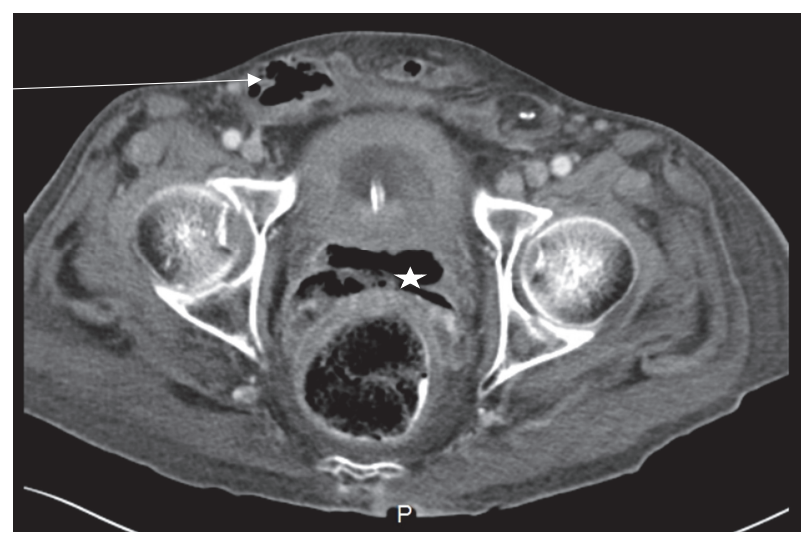

Figure 2. Computed tomography scan of the abdomen and pelvis with IV, oral and rectal contrast, axial section, showing contrast at the rectum and perineum, presence of air in the perineum, and abscess formation. Arrow showing groin abscess. Star showing air in the pelvis. count increased to 20. Urgent computed tomography (CT) scan of the abdomen was done. It showed a fistula tract between the anterior wall of the lower rectum, perineum and scrotum with multiple abscesses (Figs. $1,2,3,4,5,6)$. Lower gastrointestinal endoscopy wasn't made at the time. The patient was taken to operating room and underwent extensive debridement of the perineum and gluteal area. The abscess cavity was found to be connected to the rectal lumen through a perforation in the anterior rectal wall with bulged hard fecal mass. The team proceeded with a laparotomy and a Hartman's procedure was carried out. Also, groin abscess was incised and drained. Cultures from the abscess cavity were taken and revealed a mixed bacterial growth of gram-negative bacteria. Post operatively the patient was transferred to the intensive care unit (ICU). His sepsis was controlled and then he was transferred to the surgical floor after stabilization. He was kept on intravenous antibiotics based on culture results. The patient underwent skin grafting after resolution of the local infection.

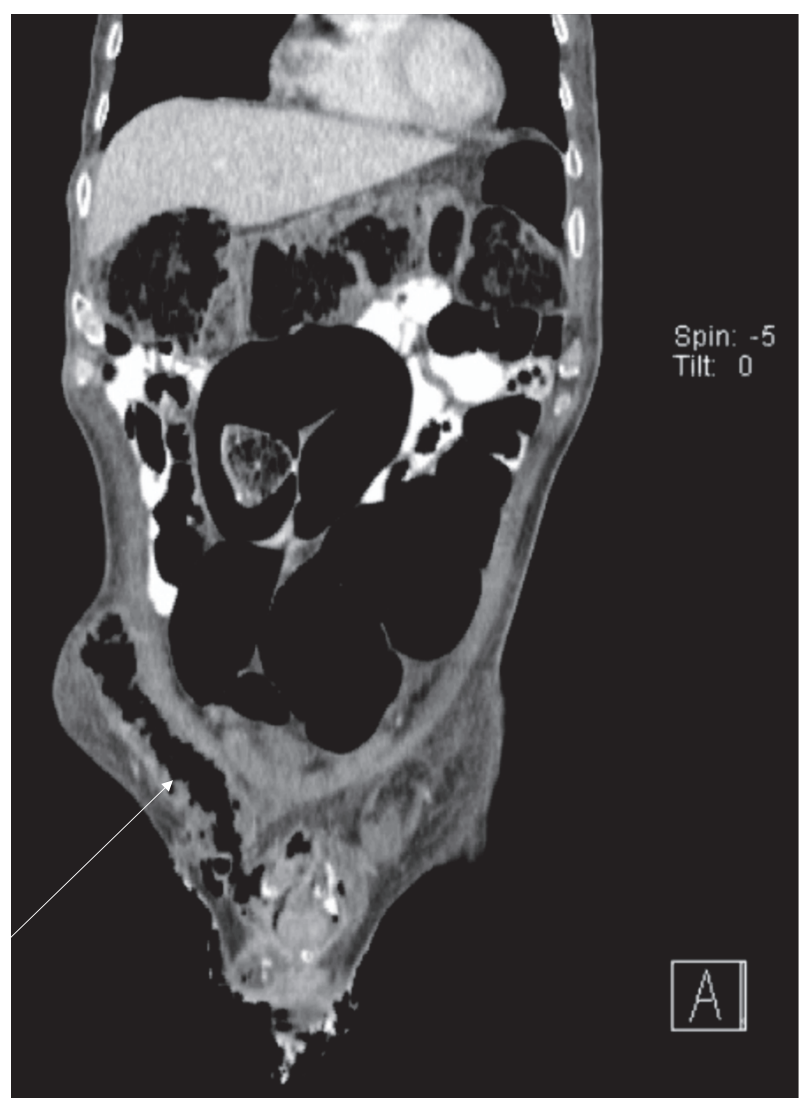

Figure 3. Computed tomography scan of the abdomen and pelvis with IV, oral and rectal contrast, coronal section, showing contrast at the rectum and perineum, presence of air in the perineum, and abscess formation. Arrow showing groin abscess. 


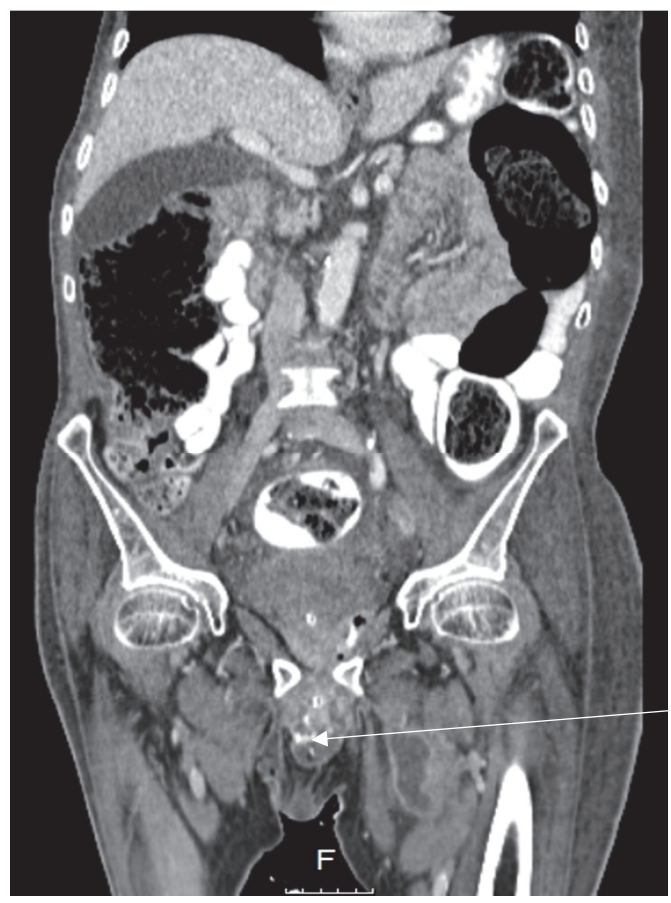

Figure 4. CT scan of the abdomen and pelvis with IV, oral and rectal contrast, Coronal section, showing contrast at the rectum and perineum, presence of air in the perineum, and abscess formation. Arrow showing contrast leak.

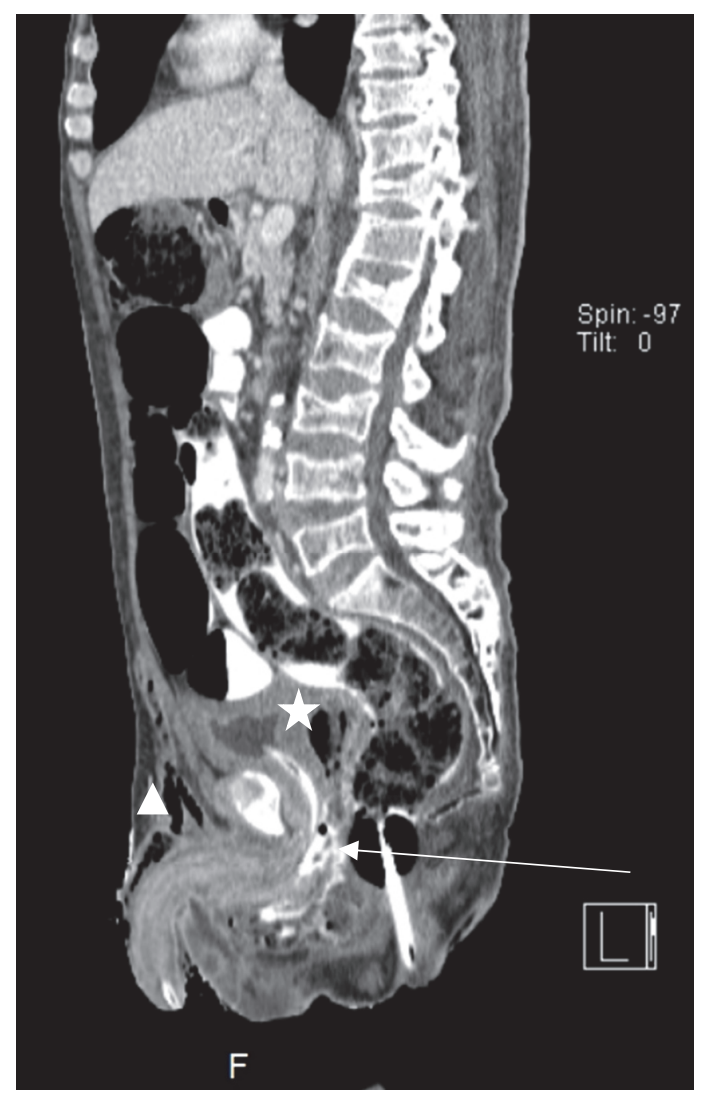

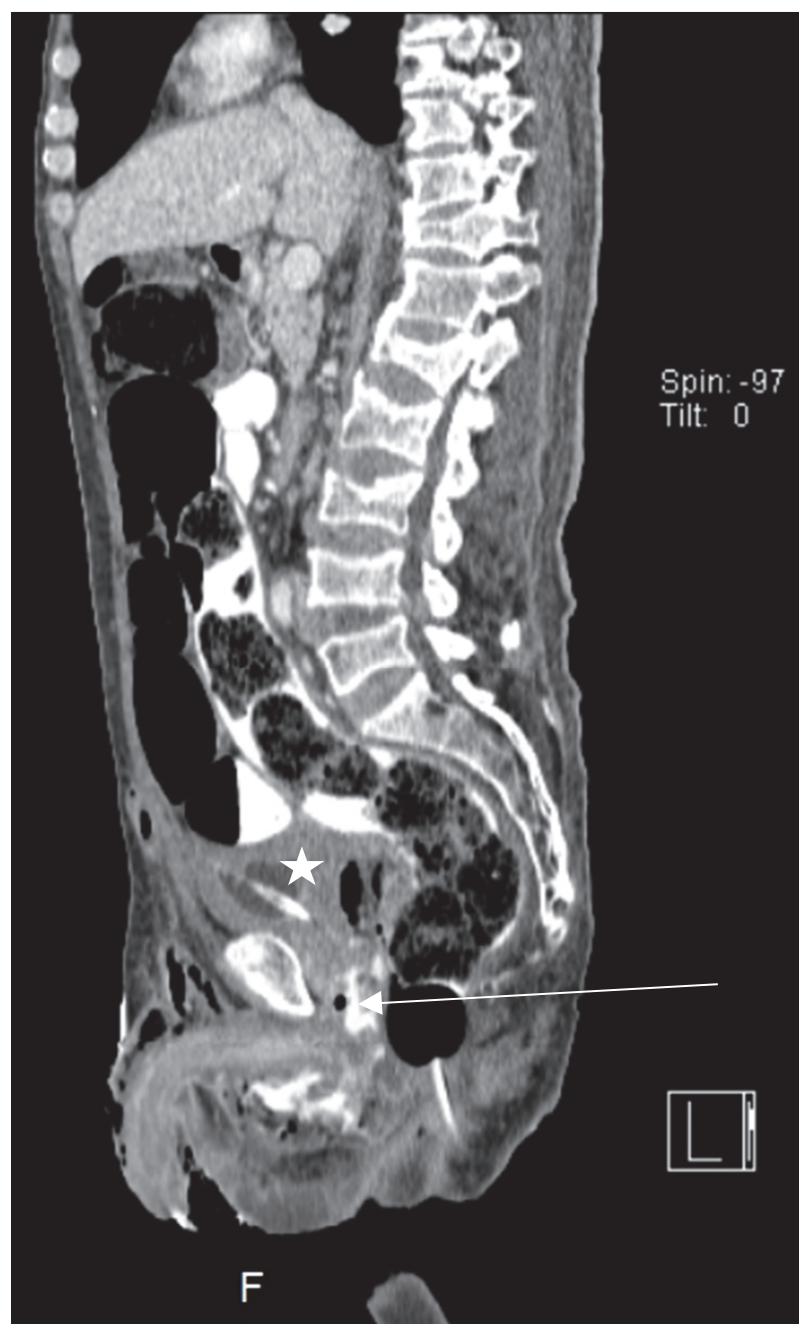

Figure 5. CT scan of the abdomen and pelvis with IV, oral and rectal contrast, Sagittal section, showing contrast at the rectum and perineum, presence of air in the perineum, and abscess formation. Arrow showing contrast leak. Star showing free air in the pelvis.

Figure 6. Computed tomography scan of the abdomen and pelvis with IV, oral and rectal contrast, sagittal section, showing contrast at the rectum and perineum, presence of air in the perineum, and abscess formation. Arrow showing contrast leak. Star showing free air in the pelvis. Triangle showing groin abscess. 


\section{Discussion}

Stercoral perforation of the rectum is an emergency situation that can be missed if not highly suspected. It is a rare disease that was first described by Berry ${ }^{[3]}$ in 1894. Around 150 cases of colonic stercoral perforation were reported in the literature ${ }^{[4]}$. Rectal involvement is even more rare with limited published data. There is no gender preference ${ }^{[5]}$.The average age of presentation is around 59 years of age ${ }^{[5]}$. Chronic constipation is the main leading cause for stercoral perforation as $81 \%$ of patients with stercoral perforation had chronic constipation ${ }^{[2]}$. Hence, the causes of chronic constipation are the risk factors of stercoral perforation ${ }^{[6]}$. The risk factors include hypothyroidism, diabetes, chronic opioid and antacid use, diabetes and neurogenic causes as Parkinson's disease all of which are associated with chronic constipation ${ }^{[6]}$. The pathology is attributed to fecaloma, which is impacted dehydrated hard fecal matter that leads to the distention of the colon and increasing the pressure on the colonic wall ${ }^{[7]}$. This increase in wall pressure compromises the blood supply to area, which results in pressure necrosis and ultimately perforation ${ }^{[7]}$. Perforation can occur at sigmoid colon, intraperitoneal or extra peritoneal part of the rectum with the sigmoid perforation being the most common ${ }^{[3]}$. The perforation is most likely to occur at the anti-mesenteric aspect as it is hypoperfused in comparison with mesenteric aspect ${ }^{[8]}$. Obviously, the left side of the colon is the commonest region in which stercoral perforation occurs. The left sided location is attributed to the narrow diameter, the lesser water content in stools, the poorer blood supply and the increasing intraluminal pressure in comparison with the right side of the colon ${ }^{[9,10]}$.

Accordingly the presenting signs and symptoms can vary ${ }^{[11]}$. Usually, it occurs in a bedbound patient with chronic constipation ${ }^{[12]}$. Patients with intraabdominal perforation usually present with abdominal pain and signs of peritonism or generalized peritonitis. Conversely, the extraperitoneal perforation of the rectum has unspecific presentation and the signs can be obscured. Rectal stercoral perforation can present as a picture of peritonitis associated with other vague symptoms including rectal discomfort, fecal incontinence and urinary symptoms ${ }^{[12]}$. While, a colonic stercoral perforation usually presents as a picture of an acute abdomen. A detailed history and examination can help in reaching the diagnosis. Imaging modalities are helpful. Chest X-ray demonstrates free air in 30\% of colonic perforation ${ }^{[7]}$. Computed tomography scan of the abdomen and pelvis is essential for early diagnosis $^{[2,7,12-14]}$. It identifies pneumoperitoneum, air in the retroperitoneum, discontinuity of bowel wall and fluid collections ${ }^{[12]}$. A high index of suspicion is required as other more common causes of colonic perforation are often thought of first such as diverticular disease, infectious and inflammatory colitis, and neoplasia ${ }^{[4]}$.

A clinical criterion was developed by Maurer et al. ${ }^{[1]}$ to help ease the diagnosis of such rare disease. The criteria includes an antimesenteric location of a more than $1 \mathrm{~cm}$ sized colonic perforation, presence of fecaloma within the colon that may extend through the perforation or within the peritoneal cavity, chronic inflammatory related changes with pressure necrosis and ulcers on microscopic exam, and other causes of perforation were eliminated.

The management of stercoral perforations should be carried out in an urgent fashion due to the high associated mortality rate ${ }^{[9]}$. The high mortality rate is usually due to sepsis or septic shock ${ }^{[9]}$. Stercoral perforations carry a higher mortality rate in comparison to other causes of colonic perforation. This is related to the co-morbidities of the patients, the older age of occurrence, the usually larger size of perforation and the higher level of intra-abdominal fecal contamination ${ }^{[14]}$. Early intervention improves the survival of patients ${ }^{[2,10]}$. Management of Stercoral perforation involves resuscitation and antibiotics with urgent surgical intervention; although, Hartman's procedure or end colostomy with rectal mucous fistula has been accepted as optimal surgical intervention ${ }^{[15]}$. Primary anastomosis with diverting loop colostomy has been described ${ }^{[8]}$. Also, primary closure of the perforation has been reported ${ }^{[10]}$. As the inflammation might involve a long bowel segment, surgeons should refrain from performing primary closure or resection with primary anastomosis ${ }^{[13]}$. The subsequent management of chronic constipation is of paramount importance.

\section{Conclusion}

Stercoral rectal perforation is a potentially fatal disease with unspecific presentations that needs a high index of suspicion in a patient with chronic constipation and severe sepsis due to an ambiguous cause. Delayed diagnosis and management may lead to catastrophic results. 


\section{Conflict of Interest}

The authors have no conflict of interest.

\section{Disclosure}

The authors did not receive any type of commercial support either in forms of compensation or financial for this study. The author has no financial interest in any of the products or devices, or drugs mentioned in this article.

\section{Ethical Approval}

Obtained.

\section{References}

[1] Maurer CA, Renzulli P, Mazzucchelli L, Egger B, Seiler CA, Büchler MW. Use of accurate diagnostic criteria may increase incidence of stercoral perforation of the colon. Dis Colon Rectum 2000; 43(7): 991-998.

[2] Chakravartty S, Chang A, Nunoo-Mensah J. A systematic review of stercoral perforation. Colorectal Dis 2013; 15(8): 930-935.

[3] BERRY J. Dilatation and rupture of sigmoid flexure [short report]. Brit Med J 1894; 1: 301.

[4] Kanwal D, Attia KME, Fam MNA, Khalil SMF, Alblooshi AM. Stercoral perforation of the rectum with faecal peritonitis and pneumatosis coli: a case report. J Radiol Case Rep 2017; 11(3): 1-6.

[5] Sharma M, Agrawal A. Case report: Stercoral sigmoid colonic perforation with fecal peritonitis. Indian J Radiol Imaging 2010; 20(2): 126-128.

[6] Obokhare I. Fecal impaction: a cause for concern? Clin Colon Rectal Surg 2012; 25(1): 53-58,

[7] Kwag SJ, Choi SK, Park JH, Jung EJ, Jung CY, Jung SH, Ju YT. A stercoral perforation of the rectum. Ann Coloproctol 2013; 29(2): 77-79.

[8] Huang WS, Wang CS, Hsieh CC, Lin PY, Chin CC, Wang JY. Management of patients with stercoral perforation of the sigmoid colon: report of five cases. World J Gastroenterol 2006; 12(3): 500-503.

[9] Edden Y, Shih SS, Wexner SD. Solitary rectal ulcer syndrome and stercoral ulcers. Gastroenterol Clin North Am 2009; 38(3): 541-545.

[10] Tokunaga Y, Hata K, Nishitai R, Kaganoi J, Nanbu H, Ohsumi K. Spontaneous perforation of the rectum with possible stercoral etiology: report of a case and review of the literature. Surg Today 1998; 28(9): 937-939.

[11] Oakenful C, Lambrianides AL. Stercoral perforation of the rectum. Emerg Med Australas 2011; 23(2): 224-227.
[12] Kumar P, Pearce O, Higginson A. Imaging manifestations of faecal impaction and stercoral perforation. Clin Radiol 2011; 66(1): 83-88.

[13] Kang J, Chung M. A stercoral perforation of the descending colon. J Korean Surg Soc 2012; 82(2): 125-127.

[14] Nam JK, Kim BS, Kim KS, Moon DJ. [Clinical analysis of stercoral perforation of the colon]. Korean J Gastroenterol 2010; 55(1): 46-51.

[15] Serpell JW, Nicholls RJ. Stercoral perforation of the colon. Br J Surg 1990; 77(12): 1325-1329. 


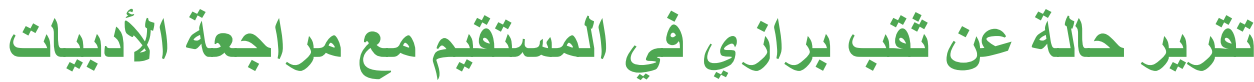

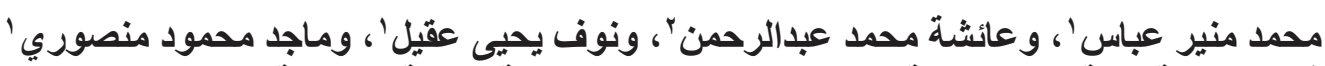

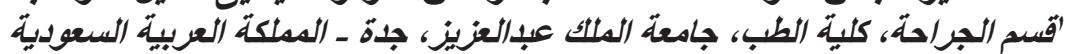

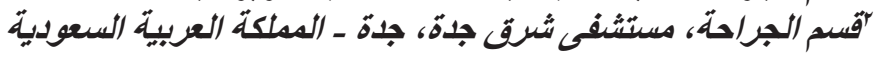

المستخلص. الثقب البرازي في المستقيم مرض نادر يسببه الإمساك المزمن. يعتبر هذا النوع من الثقوب من المضاعفات التي

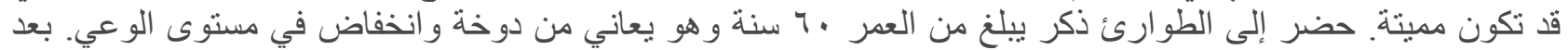

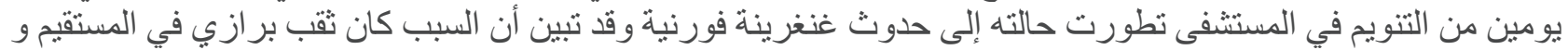

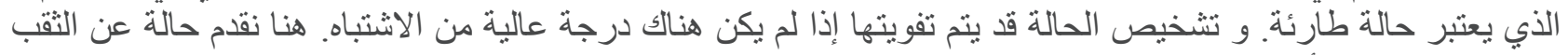

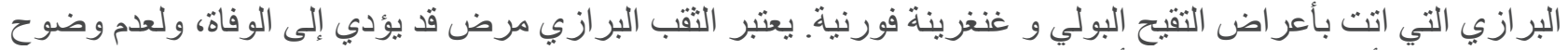

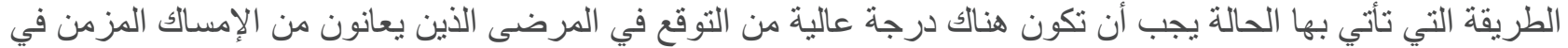
حال إصابتهم بالثقيح الثنديد في الدم. 